\title{
Expression of miR-16 is not a suitable reference for analysis of serum microRNAs in melanoma patients
}

\author{
Erica B. Friedman ${ }^{1,2}$, Shulian Shang ${ }^{3}$, Nathaniel H. Fleming ${ }^{4}$, Eleazar Vega-Saenz de Miera ${ }^{1,4}$, \\ Eva Hernando ${ }^{1,5}$, Yongzhao Shao ${ }^{1,3}$, Iman Osman, ${ }^{1,4^{*}}$
}

${ }^{1}$ Interdisciplinary Melanoma Cooperative Group, New York University, New York, USA

${ }^{2}$ Department of Surgery, New York University, New York, USA

${ }^{3}$ Division of Biostatistics, New York University, New York, USA

${ }^{4}$ Department of Dermatology, New York University, New York, USA

${ }^{5}$ Department of Pathology, New York University, New York, USA

Email: ${ }^{*}$ Iman.Osman@,nyumc.org

Received 18 September 2012; revised 20 October 2012; accepted 30 October 2012

\begin{abstract}
Background: The evolving paradigm shift towards the molecular characterization of melanoma has expanded to include studies of microRNA (miRNA) expression. As miR-16 has been utilized as a normalizer in serum-based miRNA studies in several cancers, we evaluated miR-16 expression as a potential reference for normalization of serum miRNA expression in melanoma patients. Methods: 143 primary cutaneous melanoma patients who presented to New York University (NYU) Langone Medical Center for surgical resection of AJCC stage I-III disease were studied. In addition, sera samples from 60 control subjects were utilized including 22 healthy volunteers, 13 rheumatoid arthritis patients, 20 non-melanoma cancer patients (10 renal cell carcinoma and 10 bladder cancer), and 5 Atypical Mole Syndrome patients. The KruskalWallis test $(\mathbf{k}=\mathbf{6})$ or Wilcoxon test $(\mathbf{k}=2)$ with Bonferroni correction was used for analyses of miR-16 expression in melanoma patients compared to various control groups, using raw $\mathrm{Ct}$ values directly. The Kruskal-Wallis test was used to compare miR-16 expression across stages of melanoma. The equivalence test for independent samples was used to test the equivalence of miR-16 expression among different groups. Results: No significant differential expression of miR-16 was observed between melanoma patients and healthy volunteers (Wilcoxon test, $p=0.37$ ). However, miR-16 did show a significant difference in expression as it related to stage of melanoma $(p=0.015)$. Additionally, the equivalence test was unable to confirm equivalent expression of miR-16 in any melanoma versus control group pair. Conclusion: Our data indicate that miR-16 cannot be used as a universal normalizer in sera studies of melanoma patients.
\end{abstract}

"Corresponding author.
Keywords: MicroRNA; Melanoma; Normalization; Reference Gene

\section{INTRODUCTION}

In the era of personalized medicine, the identification of molecular markers with utility in aiding diagnosis, assessing prognosis, and monitoring for recurrence of tumors is critical. As regulatory biomolecules with roles in fundamental cellular processes, including cancer [1,2], microRNAs (miRNAs) have emerged as important targets in the search for new tumor markers. Due to ease of collection, the search for clinically useful biomarkers has lead to exploration of the potential of serum-based microRNAs.

Innovative adaptations of existing technologies, specifically quantitative reverse transcription-PCR (qRTPCR), have helped to overcome the challenge of detecting and quantifying theses small non-coding RNAs (18 25 nucleotides). However, an effective normalization strategy to control sample-to-sample variations introduced by specimen quality and protocol inconsistencies (i.e. extraction yield, RT yield, efficiency of amplification) remains a challenge. Traditionally, non-biological variations have been corrected by using internal reference genes, or "housekeeping genes" [3]. However, it is unlikely that a single universal reference gene for all tissue types exists [3] and if inappropriately selected, could lead to errors and misinterpretation of the data [4]. Normalization is a particularly difficult challenge in the study of circulating miRNAs, as the source of these miRNAs is as yet undetermined, making the application of established tissue based reference genes insufficient, and there are few reports of validated controls in serum or plasma [5].

Though there is no current consensus on suitable references for analysis of serum miRNAs, miR-16 was re- 
ported to be relatively invariant across a large number of samples [6] and is often selected as a stable control for normalization [7]. However, data to support the suitability of miR-16 as a reference gene for qRT-PCR analysis of blood-based miRNAs are limited [8,9]. Here, we evaluate miR-16 as a potential reference in the study of serum-based miRNAs in melanoma patients.

\section{PATIENTS AND METHODS}

\subsection{Patient Enrollment and Blood Collection}

Blood samples were obtained from melanoma patients treated at New York University (NYU) Langone Medical Center who signed informed consent prior to surgery for inclusion into the Interdisciplinary Melanoma Cooperative Group [10]. Tumor specimens from all patients undergo centralized histopathological review to confirm a diagnosis of melanoma. Control samples were collected from healthy volunteers, defined as individuals with no current or past diagnosis of cancer or systemic inflammatory condition, rheumatoid arthritis patients with active disease, non-melanoma cancer patients with stage IV renal cell carcinoma or bladder cancer, and Atypical Mole Syndrome patients, defined by previously identified criteria [11] and with no personal history of melanoma. The NYU and Memorial Sloan-Kettering Cancer Center (MSKCC) Institutional Review Board approved the study and informed consent was obtained from all patients.

All melanoma and control blood samples intended for serum preparation, with the exception of non-melanoma cancer patients, were collected and processed under standard conditions at NYU. Non-melanoma cancer patient samples were collected and processed at MSKCC under the same conditions. Specifically, blood was collected in 10-mL BD no additive serum tubes (catalog \#: 367820 ), stored immediately at $4^{\circ} \mathrm{C}$ for up to 2 hours and then centrifuged at $10^{\circ} \mathrm{C}$ for 10 minutes at $1500 \mathrm{~g}$. The supernatant serum was then aliquoted into $1.5 \mathrm{~mL}$ cryovials and stored at $-80^{\circ} \mathrm{C}$ until further use.

All samples utilized for miRNA extraction and subsequent analyses of miRNA expression levels were subjected to a single freeze-thaw cycle. Total RNA from $1 \mathrm{ml}$ of serum was extracted and purified using the miRVana Paris isolation system (Ambion, USA) following the manufacturer's protocol with the addition of an extra $\mathrm{acid} / \mathrm{phenol} / \mathrm{chloroform}$ extraction. RNA was eluted with $100 \mu \mathrm{l}$ of RNase free water.

\subsection{Reverse Transcription Methods}

RT was performed following manufacturer's instructions using TaqMan MicroRNA Reverse Transcription kit (Applied Biosystems, USA) using $2.5 \mu$ l total RNA input in a final volume of $10 \mu \mathrm{l}$ using TaqMan MicroRNA Reverse Transcription kit following manufacturer's instructions. Quantitative PCR was performed in triplicate on a Bio-Rad MyIQ Single Color Real Time PCR detection system. Each amplification reaction was performed using $1.33 \mu \mathrm{l}$ of the cDNA, $1 \mu \mathrm{l}$ of miR-16-specific TaqMan ${ }^{\circledR}$ primer and $1 \mathrm{X}$ Hotmaster Master mix containing Taq DNA polymerase and dNTP Mix in a final volume of 20 $\mu l$.

\subsection{Statistical Methods}

The Kruskal-Wallis test $(\mathrm{k}=6)$ or Wilcoxon test $(\mathrm{k}=2)$ with Bonferroni correction was used for analyses of miR-16 expression, using raw $\mathrm{Ct}$ values directly, in melanoma patients compared to various control groups. Kruskal-Wallis test was used to compare miR-16 expression across stages of melanoma. An alpha at $\alpha=0.05$ was used to determine significance for all tests. After log transformation of $\mathrm{Ct}$ values, the previously described equivalence test for independent samples was used to test equivalence of miR-16 expression among different groups [12]. A cutoff of fold change $\geq 2$ was selected to set the deviation area $[-1 ; 1]$. All statistical analyses were performed using R 2.12.1.

\section{RESULTS}

A total of 143 primary cutaneous melanoma patients who presented to New York University (NYU) Langone Medical Center for surgical resection of AJCC stage I-III disease were studied (Table 1). Sera samples from 60 control subjects were utilized and included 22 healthy volunteers, 13 rheumatoid arthritis patients, 20 nonmelanoma cancer patients (10 renal cell carcinoma and 10 bladder cancer), and 5 Atypical Mole Syndrome patients (Table 2).

The expression level of miR-16 was analyzed by qRTPCR in serum samples from melanoma patients and controls. When miR-16 expression was compared across all groups (Figure 1(a)), there was a significant difference in at least one of the groups, detectable by the KruskalWallis one-way analysis of variance $(p=0.05)$. Upon further analysis of all possible two-group comparisons using the Wilcoxon test, there was a significant difference in expression levels of miR-16 in rheumatoid arthritis compared to bladder cancer after Bonferroni correction $(p=0.019)$. Notably, there was no evidence of significant differential expression of miR-16 between melanoma patients and healthy volunteers $(p=0.37)$, which would lend support for its potential as a reference miRNA in diagnostic studies. However, when sera miR-16 expression was evaluated within melanoma patients, we found a significant difference in expression as it related to stage (Kruskal-Wallis test, $\mathrm{p}=0.015$, Figure 1(b)). 
Table 1. Baseline characteristics of melanoma patients.

\begin{tabular}{|c|c|}
\hline & Melanoma patients $(n=143) \mathrm{N}(\%)$ \\
\hline \multicolumn{2}{|l|}{ Age at diagnosis, years } \\
\hline Median & 57 \\
\hline \multicolumn{2}{|l|}{ Gender } \\
\hline Male & $82(57)$ \\
\hline Female & $61(43)$ \\
\hline \multicolumn{2}{|l|}{ Stage } \\
\hline I & $49(34)$ \\
\hline II & $65(45)$ \\
\hline III & $29(20)$ \\
\hline \multicolumn{2}{|l|}{ Thickness, mm } \\
\hline Range & $0.12-28$ \\
\hline \multicolumn{2}{|l|}{ Ulceration } \\
\hline Present & $49(34)$ \\
\hline Absent & $94(66)$ \\
\hline \multicolumn{2}{|l|}{ Mitosis } \\
\hline Absent & $30(21)$ \\
\hline Present & $75(52)$ \\
\hline Unclassified & $38(27)$ \\
\hline \multicolumn{2}{|l|}{ Histological type } \\
\hline Superficial Spreading & $50(35)$ \\
\hline Nodular & $42(29)$ \\
\hline Other $^{1}$ & $14(10)$ \\
\hline Unclassified & $37(26)$ \\
\hline
\end{tabular}

Note: Percentages may not sum to $100 \%$ as a result of rounding; ${ }^{1}$ Other includes acral lentiginous melanoma, desmoplastic melanoma and lentigo maligna.

While the Wilcoxon test, and its parametric counterpart, the Student's t-test, are appropriate for the identification of differentially expressed target genes, they are not well suited for validation of stable expression. Therefore, we performed the equivalence test [12] to show additional support that miR-16 is not a good candidate reference gene for normalization in serum-based miRNA studies in melanoma. A fold change of $\geq 2$ was selected to define a significant difference in expression, corresponding to a deviation area $[-1 ; 1]$ for the logarithmized expression. When comparing melanoma samples to each of the control groups, equivalent expression of miR-16 was not confirmed in any melanoma/ control group pair (Figure 1(c)). miR-16 showed a tendency for higher expression in melanoma compared to rheumatoid arthritis, whereas it appeared to be more highly expressed in healthy volunteers, renal cell carcinoma, bladder cancer and Atypical Mole Syndrome than melanoma.

\section{DISCUSSION}

As the purpose of normalization is to remove all variation that is not a consequence of the disease itself [13], an ideal reference gene must not only exhibit invariant expression across all sample groups, but also be stably expressed throughout all stages, grades and subtypes of a disease. Our data demonstrate that miR-16 shows significantly different expression across stages of melanoma, suggesting that if used as a normalizer, obtained results might be entirely incorrect. Additionally, while Wilcoxon tests supported non-differential expression of miR-16 across a diverse population, the equivalence test fails to establish equivalence of miR-16 expression in melanoma patients compared to each of the control groups. To the best of our knowledge, this is the first report to evaluate miR-16 as a reference gene for the study of serum miRNA studies in melanoma.

miRNA stability in serum can be partially explained by their physical association with lipoprotein complexes, including small membrane vesicles of endocytic origin called exosomes or microvesicles $(30-100 \mathrm{~nm})$ [14]. Importantly, exosomes represent a newly discovered mechanism by which donor cells can communicate and influence the gene expression of recipient cells $[15,16]$. Nevertheless, little is known about the mechanisms by which miRNAs are generated in plasma and the biologic impact of these molecules in distant sites of the body [5]. Growing evidence indicates that exosomal miRNA packaging occurs non-randomly based on differential expression of exosomal miRNA compared to that of donor cells. Indeed, studies have demonstrated that nearly $30 \%$ of the released miRNAs in vitro and in vivo do not reflect the expression profile found in donor cells, suggesting that specific miRNAs are selected to be intracellularly retained or released by exosomes [17]. In order to successfully study these circulating molecules, it is imperative to select the correct reference gene for normalization.

Finally, RNU6B, frequently used as a reference gene in tissue based mRNA and miRNA studies, is not consistently detected in plasma or serum. This is not surprising as this RNA is nuclear and, as such, is not protected by extracellular membranes. In our experience, we fail to detect RNU6B in all the control sera and in most of the other sera used for this paper. Although there is least one report of the use of RNU6B in sera [18], there are also results with sera in which this small RNA is unstable, cannot be detected or Cts are high and unreliable $[19,20]$. 
Table 2. Baseline characteristics of control patients.

\begin{tabular}{|c|c|c|c|c|c|}
\hline & $\begin{array}{l}\text { Healthy Volunteers } \\
\qquad(\mathrm{n}=22)\end{array}$ & $\begin{array}{l}\text { Rheumatoid Arthritis } \\
\qquad(\mathrm{n}=13)\end{array}$ & $\begin{array}{l}\text { Bladder Cancer } \\
\quad(\mathbf{n}=\mathbf{1 0})\end{array}$ & $\begin{array}{l}\text { Renal Cell Carcinoma } \\
\qquad(\mathrm{n}=\mathbf{1 0})\end{array}$ & $\begin{array}{r}\text { Dysplastic Nevus } \\
\text { Syndrome }(n=5)\end{array}$ \\
\hline \multicolumn{6}{|l|}{ Gender N (\%) } \\
\hline Male & $9(41)$ & $4(31)$ & $6(60)$ & $7(70)$ & $4(80)$ \\
\hline Female & $13(59)$ & $9(69)$ & $4(40)$ & $3(30)$ & $1(20)$ \\
\hline \multicolumn{6}{|l|}{ Age, years } \\
\hline Median (Range) & $31.5(22-59)$ & $41(32-67)$ & $64.5(42-75)$ & $59.5(48-70)$ & $39(27-51)$ \\
\hline \multicolumn{6}{|l|}{ Stage N (\%) } \\
\hline Stage IV & N/A & N/A & $10(100)$ & $10(100)$ & N/A \\
\hline \multicolumn{6}{|l|}{ DAS28 } \\
\hline Median (Range) & N/A & $6.68(3.62-7.82)$ & N/A & N/A & N/A \\
\hline
\end{tabular}

DAS28: Disease Activity Score for Rheumatoid Arthritis.

miR. 16

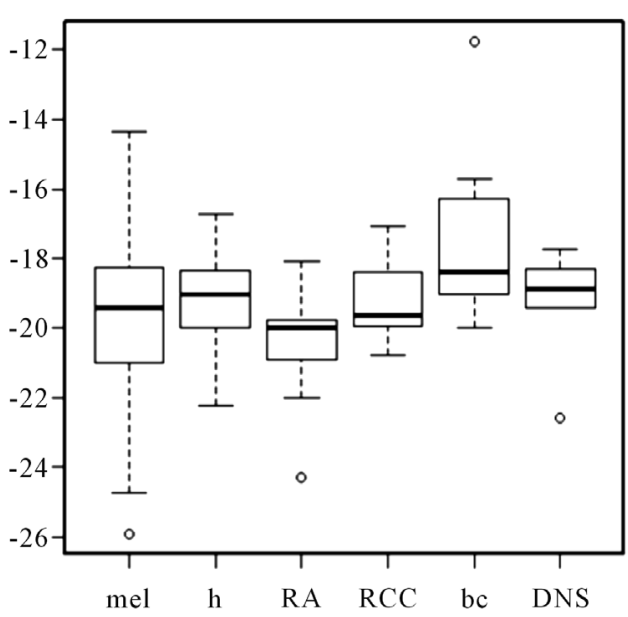

(a)

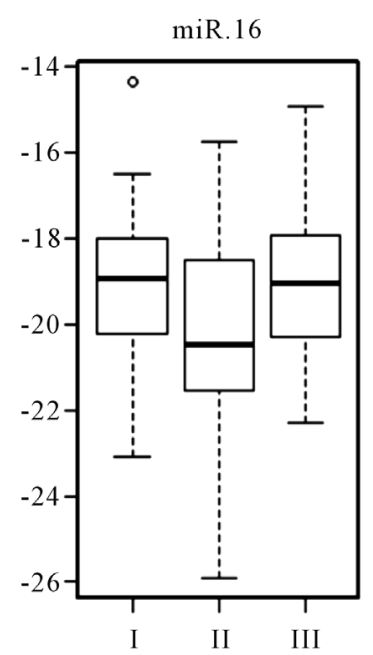

(b)

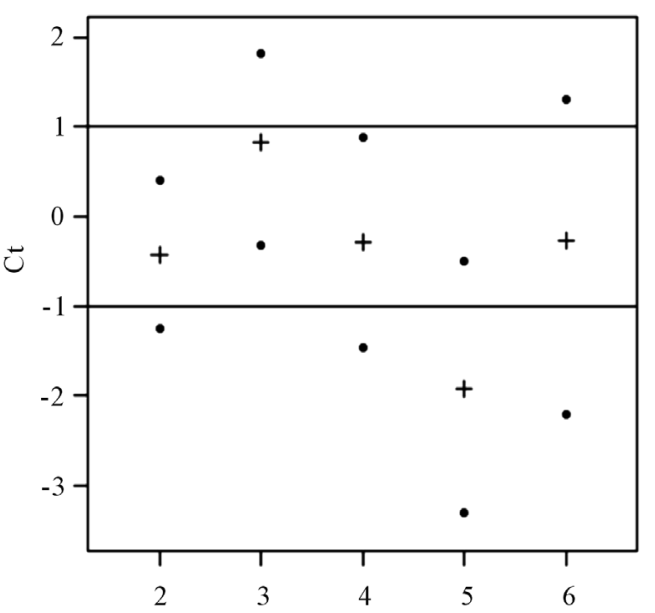

(c)

Figure 1. miR-16 expression in melanoma and control samples. (a) miR-16 expression (-Ct) in melanoma patients, healthy volunteers (healthy), Rheumatoid Arthritis (RA), renal cell carcinoma (RCC), bladder cancer (BC) and Atypical Mole Syndrome (AMS) patients as measured by qRT-PCR; (b) miR-16 expression of stage I-III melanoma patients was significantly different by the Kruskal-Wallis test $(\mathrm{p}=0.015)$; (c) Equivalence test for miR-16 in melanoma compared to the listed control groups. Shown are differences of the means $(+)$ and confidence intervals $(\bullet)$ for the logarithmized relative expression of miR-16. The deviation area $[-1 ; 1]$ for a fold change $\leq 2$ is plotted as continuous lines. Since none of the confidence intervals fall within the deviation area, miR-16 is not shown to be equivalently expressed in any of the melanoma/control pairs.

\section{CONCLUSION}

Our data indicate that miR-16 is not a suitable reference miRNA for qRT-PCR analysis of serum miRNA in melanoma patients. To ensure detection of small, yet biologically meaningful changes and avoid misinterprettation of results, further testing is needed to identify suitable candidates for normalization in miRNA-based melanoma studies.

\section{ACKNOWLEDGEMENTS}

The authors thank Steven B. Abramson, M.D., Jeffrey D. Greenberg, M.D., and Mukundan Attur, Ph.D. (NYU School of Medicine/NYU Hospital for Joint Disease, New York, NY) for providing the rheumatoid arthritis patient sera samples; Dean F. Bajorin, M.D. (Memorial Sloan-Kettering Cancer Center, New York, NY) for providing the renal cell carcinoma and bladder cancer sera samples; and David Polsky, M.D., Ph.D. and Jennifer Stein, M.D. (NYU School of Medicine, De- 
partment of Dermatology, New York, NY) for providing the Atypical Mole Syndrome patients. We also thank Michelle Ma, Daniel Lackaye and Holly Greenwald for the management of the clinical information of the IMCG patients. All authors have read the journal's policy on disclosure of potential conflicts of interest and have nothing to disclose at this time. This research is supported in part by: Grant 1UL1RR029893 from the National Center for Research Resources, National Institutes of Health, NYU Cancer Institute Cancer Center Support Grant 5P30CA16087-27, and the Marc Jacobs Campaign to support the Interdisciplinary Melanoma Cooperative Group.

\section{REFERENCES}

[1] Lu, J., Getz, G., Miska, E.A., et al. (2005) MicroRNA expression profiles classify human cancers. Nature, $\mathbf{4 3 5}$, 834-838. doi:10.1038/nature03702

[2] McManus, M.T. (2003) MicroRNAs and cancer. Seminars in Cancer Biology, 13, 253-258. doi:10.1016/S1044-579X(03)00038-5

[3] Vandesompele, J., De Preter, K., Pattyn, F., et al. (2002) Accurate normalization of real-time quantitative RT-PCR data by geometric averaging of multiple internal control genes. Genome Biology, 3, RESEARCH0034.

[4] Dheda, K., Huggett, J.F., Chang, J.S., et al. (2005) The implications of using an inappropriate reference gene for real-time reverse transcription PCR data normalization. Analytical Biochemistry, 344, 141-143. doi:10.1016/j.ab.2005.05.022

[5] Cortez, M.A. and Calin, G.A. (2009) MicroRNA identification in plasma and serum: A new tool to diagnose and monitor diseases. Expert Opinion on Biological Therapy, 9, 703-711. doi:10.1517/14712590902932889

[6] Kroh, E.M., Parkin, R.K., Mitchell, P.S., et al. (2010) Analysis of circulating microRNA biomarkers in plasma and serum using quantitative reverse transcription-PCR (qRT-PCR). Methods, 50, 298-301. doi:10.1016/j.ymeth.2010.01.032

[7] Cortez, M.A., Bueso-Ramos, C., Ferdin, J., et al. (2011) MicroRNAs in body fluids-The mix of hormones and biomarkers. Nature Reviews: Clinical Oncology, 8, 467477.

[8] Song, J.N., Bai, Z.G., Han, W., et al. (2012) Identification of suitable reference genes for qPCR analysis of serum microRNA in gastric cancer patients. Digestive Diseases and Sciences, 57, 897-904. doi:10.1007/s10620-011-1981-7

[9] Zhu, H.-T., Dong, Q.-Z., Wang, G., et al. (2012) Identification of suitable reference genes for qRT-PCR analysis of circulating microRNAs in hepatitis $\mathrm{B}$ virus-infected patients. Molecular Biotechnology, 50, 49-56. doi:10.1007/s12033-011-9414-6

[10] Wich, L.G., Hamilton, H.K., Shapiro, R.L., et al. (2009) Developing a multidisciplinary prospective melanoma biospecimen repository to advance translational research. American Journal of Translational Research, 1, 35-43.

[11] Kopf, A.W., Friedman, R.J. and Rigel, D.S. (1990) A typical mole syndrome. Journal of the American Academy of Dermatology, 22, 117-118. doi:10.1016/S0190-9622(08)80006-0

[12] Haller, F., Kulle, B., Schwager, S., et al. (2004) Equivalence test in quantitative reverse transcription polymerase chain reaction: Confirmation of reference genes suitable for normalization. Analytical Biochemistry, 335, 1-9. doi:10.1016/j.ab.2004.08.024

[13] Peltier, H.J. and Latham, G.J. (2008) Normalization of microRNA expression levels in quantitative RT-PCR assays: Identification of suitable reference RNA targets in normal and cancerous human solid tissues. RNA, 14, 844852. doi: $10.1261 /$ rna. 939908

[14] Hunter, M.P., Ismail, N., Zhang, X.L., et al. (2008) Detection of microRNA expression in human peripheral blood microvesicles. PLoS ONE, 3, e3694. doi:10.1371/journal.pone.0003694

[15] Valadi, H., Ekström, K., Bossios, A., et al. (2007) Exosome-mediated transfer of mRNAs and microRNAs is a novel mechanism of genetic exchange between cells. $\mathrm{Na}$ ture Cell Biology, 9, 654-659. doi:10.1038/ncb1596

[16] Skog, J., Wurdinger, T., Van Rijn, S., et al. (2008) Glioblastoma microvesicles transport RNA and proteins that promote tumour growth and provide diagnostic biomarkers. Nature Cell Biology, 10, 1470-1476. doi:10.1038/ncb1800

[17] Pigati, L., Yaddanapudi, S.C.S., Iyengar, R., et al. (2010) Selective release of microRNA species from normal and malignant mammary epithelial cells. PLoS ONE, 5, e13515. doi:10.1371/journal.pone.0013515

[18] Ng, E.K., Chong, W.W., Jin, H., et al. (2009) Differential expression of microRNAs in plasma of patients with colorectal cancer: A potential marker for colorectal cancer screening. Gut, 58, 1375-1381. doi:10.1136/gut.2008.167817

[19] Shen, J., Todd, N.W., Zhang, H., et al. (2011) Plasma microRNAs as potential biomarkers for non-small-cell lung cancer. Laboratory Investigation, 91, 579-587. doi:10.1038/labinvest.2010.194

[20] Huang, Z.H., Huang, D., Ni, S.J., et al. (2010) Plasma microRNAs are promising novel biomarkers for early detection of colorectal cancer. International Journal of Cancer, 127, 118-126. doi:10.1002/ijc. 25007 